\title{
Migration on the borderline
}

During epithelial-to-mesenchymal transitions or cell migration, there must be changes in gene expression, cell-cell adhesion and actin organization. These processes are essential for embryonic development, but can also lead to tumour metastasis, so it is imperative that we understand the mechanisms behind this transition. The border cells in the Drosophila ovary are often used as a simple model to study such cell migration. Border cells are a group of six to ten cells that delaminate from the anterior follicle cells and migrate over the germline cells towards the posterior of the egg chamber. They stop migrating when they reach the anterior edge of the oocyte. Many genes have now been identified that function in controlling the migration of these cells, such as the basic-region/leucine zipper transcription factor C/EBP homologue, slow border cells (slbo) and DE-cadherin.

A recent study by Liu and Montell (Development 128, 321-330; 2001) has now uncovered a new player in this process, jing, which encodes the Drosophila homologue of the mouse protein AEBP2. AEBP2 is a proposed transcriptional repressor that binds a small sequence within the adipocyte AP2 gene, to which the mammalian homologue of slbo also binds. Jing is a nuclear factor that, like Slbo, is required for initiation of border cell migration. In jing-mutant egg chambers, the border cells do not move towards the oocyte, but remain at the anterior end of the chamber (see picture; bc, border cells; on, oocyte nucleus). Expression of jing is dependent on the presence of slbo, but more interestingly, overexpression of jing can rescue some of the defects associated with reduced Slbo levels,

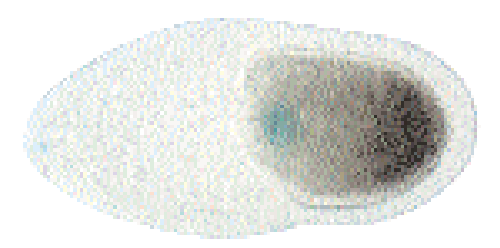

IMAGE COURTESY OF THE COMPANY OF BIOLOGISTS

including border-cell migration in some slbo mutants.

As C/EBP and AEBP2 bind to similar regions within mammalian AP2, it seems highly likely that both Slbo and Jing will interact in a similar manner to regulate the expression of genes that are essential for border-cell migration. As adipocytes and border cells coordinate their differentiation with the nutritional wellbeing of the organism, it seems that both processes may also require a hormonal signal for the actions of slbo/C/EBP and jing/AEBP2 to be initiated. However, we have no idea of the nature of this signal or of the identities of most of the downstream genes activated by slbo and jing. So, although identifying jing as an important gene in border-cell migration is a step in the right direction, we are still a few more genes away from understanding how this important transition is activated.

SARAH GREAVES 